\title{
Successful osimertinib rechallenge following subsequent chemotherapy regimen in a patient with metastatic non-small cell lung carcinoma: a case report
}

\author{
Zhao-Jie $\mathrm{Han}^{1}$, Nuo $\mathrm{Luo}^{2}, \mathrm{Li} \mathrm{Li}^{2}$, Zhu-Lin Liu ${ }^{2}$ \\ ${ }^{1}$ Department of Thoracic Surgery, Southwest Hospital, Army Medical University, Chongqing, China; ${ }^{2}$ Department of Respiratory Disease, Daping \\ Hospital, Army Medical University, Chongqing, China \\ Correspondence to: Zhu-Lin Liu. Department of Respiratory Disease, Daping Hospital, Army Medical University, Chongqing 400042, China. \\ Email: 624927883@qq.com.
}

\begin{abstract}
Although tyrosine kinase inhibitors (TKIs) targeting epidermal growth factor receptor (EGFR) have a favorable and durable treatment response, almost all patients will eventually acquire resistance and develop disease progression. Re-administration of first and second-generation EGFR TKIs has been successfully executed in advanced non-small cell lung cancer (NSCLC) subsequent to EGFR-TKI resistance. However, osimertinib rechallenge following osimertinib resistance in EGFR T790M-negative patient is less explored. Herein, we describe a metastatic adenocarcinoma NSCLC patient with exon 19 deletion in EGFR (19del) who acquired resistance to initial gefitinib and second-line osimertinib but was successfully rechallenged with osimertinib following treatment failure with chemotherapy. The osimertinib rechallenge, despite the absence of EGFR T790M, was considered after the development of multiple small pulmonary lesions and an increase in EGFR exon 19 deletion. After a month of osimertinib rechallenge, pulmonary and brain lesions significantly reduced achieving partial response. The success of osimertinib rechallenge following previous osimertinib resistance in a metastatic NSCLC patient with EGFR 19del in the absence of T790M suggests that re-administration of osimertinib can be a treatment option in similar situations. In addition, this case also highlights the importance of mutational profiling for treatment monitoring to understand the mutational landscape of the patient and guide subsequent treatment including treatment rechallenge.
\end{abstract}

Keywords: Epidermal growth factor receptor-tyrosine kinase inhibitors (EGFR-TKI); osimertinib resistance; osimertinib rechallenge; CCDC6-RET fusion

Submitted Nov 29, 2020. Accepted for publication Feb 24, 2021.

doi: 10.21037/apm-20-2369

View this article at: http://dx.doi.org/10.21037/apm-20-2369

\section{Introduction}

Epidermal growth factor receptor (EGFR) tyrosine kinase inhibitors (TKI) have dramatically improved the survival of $E G F R$-mutant adenocarcinoma non-small cell lung cancer (NSCLC) patients (1). Despite the significant improvement in survival outcomes, almost all patients eventually acquire resistance and develop disease progression (2). A significant percentage of acquired resistance involves secondary mutations in EGFR, including T790M (3). Osimertinib, a third-generation EGFR-TKI, irreversibly and preferentially binds to mutated receptors and is the treatment of choice for patients who have EGFR T790M-mediated resistance $(4,5)$. Recently, FLAURA clinical trial has revealed that osimertinib has superior efficacy than first and secondgeneration EGFR-TKI for treatment-naïve EGFR-mutant patients, extending its utilization as first-line therapy (6). After treatment failure with osimertinib, platinum-based chemotherapy is often the standard of care (7). However, no consensus exists on further treatment strategies on subsequent treatment failures. Under the premise that 
subsequent lines of therapy could potentially kill resistant clones and allow the regrowth of inhibitor-sensitive clones, a potential strategy after acquiring resistance to preceding lines of therapy is the re-administration of EGFR-TKI (8). Re-administration, also termed as rechallenge, of first and second-generation EGFR-TKIs has been successful and well-documented in NSCLC patients (8-12), with disease control rate of up to $85.2 \%$ and median progression-free survival (PFS) of up to 6 months (8). However, there is limited information regarding osimertinib rechallenge succeeding chemotherapy regimen following subsequent osimertinib resistance. Currently, only five cases have reported on the success of osimertinib rechallenge in EGFR T790M-positive NSCLC patients following osimertinib resistance $(13,14)$. Other reports on successful osimertinib rechallenge were regarding treatment re-administration subsequent to osimertinib-induced adverse events (15-17). In this study, we report the efficacy of osimertinib rechallenge in an EGFR 19del-positive T790M-negative metastatic NSCLC patient.

We present the following article in accordance with the CARE reporting checklist (available at http://dx.doi. org/10.21037/apm-20-2369).

\section{Case presentation}

A 50-year-old male smoker was referred to our clinic due to persistent productive cough for 2 months. Initial chest computed tomographic (CT) scans revealed a mass with a maximum diameter of $7.8 \mathrm{~cm}$ on the right upper lobe of the lung and presence of multiple nodules in both lungs suggesting extensive lung metastasis and lymph node involvement (Figure 1). Magnetic resonance imaging (MRI) of the brain did not reveal any lesions. Bone scans revealed multiple bone metastases. The patient was diagnosed as having T4N2M1b, stage IV adenocarcinoma. The patient scored 0 for Eastern Cooperative Oncology Group (ECOG) performance status (PS). Further immunohistochemistry staining revealed negative for ALK, ROS-1, PD-1, and PDL1, while positive for TTF-1 and Ki-67 (3\%). Gefitinib at a dose of $250 \mathrm{mg}$ once daily was administered after identification of an EGFR exon 19 deletion (19del). After 5 months of treatment, a remarkable reduction of the primary tumor was observed, achieving partial response (PR) based on response evaluation criteria in solid tumors (RECIST v1.1). After 7 months of gefitinib, the patient developed new brain lesions and the primary lung lesion enlarged, assessed as disease progression (PD). Capture- based targeted sequencing performed on his plasma sample at PD revealed EGFR T790M. The circulating tumor DNA were isolated from the patient blood samples using Qiagen DNA isolation kits, and processed for subsequent NGS library construction using optimized protocols. Sequencing was performed using Illumina NextSeq 500 using paired-end reads with target sequencing depth of 10,000X. The sequencing data were analyzed using bioinformatics pipeline optimized for somatic variant calling. Target capture was performed using commercially available panel consisting of 168 lung cancer-related genes (Figure 2). Accordingly, osimertinib at a dosage of $80 \mathrm{mg}$ once daily was administered. Within 2 months of osimertinib therapy, chest CT and brain MRI revealed drastic tumor reduction achieving an overall response of $\mathrm{PR}$ accompanied by significant reduction in allele frequency (AF) of the genomic alterations, including the disappearance of EGFR T790M. Unfortunately, after 7 months of osimertinib therapy, the patient again developed chest pain. Chest CT showed an enlargement of the lung lesions (Figure $1 B$ ). Targeted sequencing revealed a previously undetected CCDC6-RET fusion and gene amplifications in KRAS, VEGFA, and CSMD3. The patient was placed on pemetrexed, cisplatin and bevacizumab regimen. The patient refused to undergo radiotherapy for brain lesions. Albeit improvement of the patient's clinical symptoms after 2 cycles of the treatment, no significant reduction in the lung lesions was observed (Figure 1C), achieving stable disease (SD). After a total of 4 cycles of chemotherapy, chest CT revealed the development of multiple small pulmonary lesions, indicating PD (Figure 1D). In addition, sequencing data revealed an increase in the AF of EGFR $19 \mathrm{del}(22.41 \%)$, indicating a possible resensitization with EGFR-TKI. Although undetected, the presence of EGFR T790M cannot be completely ruled out. Hence, a rechallenge with osimertinib was considered. Osimertinib was administered within 1 week after chemotherapy with a dosage of $80 \mathrm{mg}$ once daily. Significant reduction in lung and brain lesions were observed after 1 month of osimertinib re-administration (Figure $1 E$ ) for scans of the lung lesions; data not shown for brain lesions), achieving PR as best response. On September 2018, with a PFS of 4 months, the patient was assessed as PD after complaints of neurologic decline such as slurred speech and weakness in the legs leading to difficulty in walking.

All procedures performed in studies involving human participants were in accordance with the ethical standards of the institutional and/or national research committee(s) and 
A
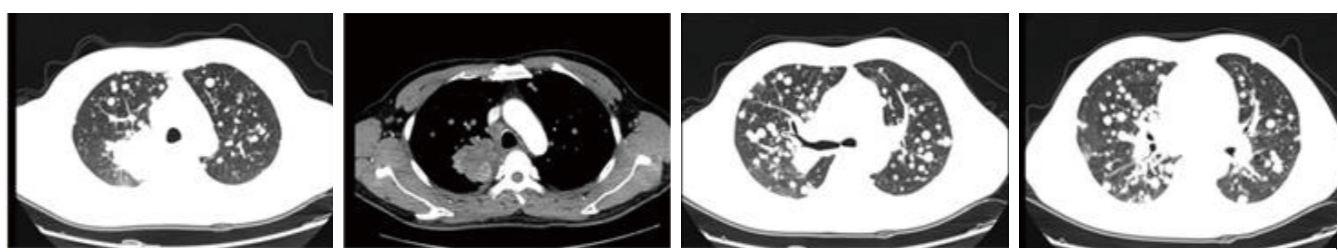

B

7 months after osimertinib therapy, before chemotherapy January 2018 (status: PD)
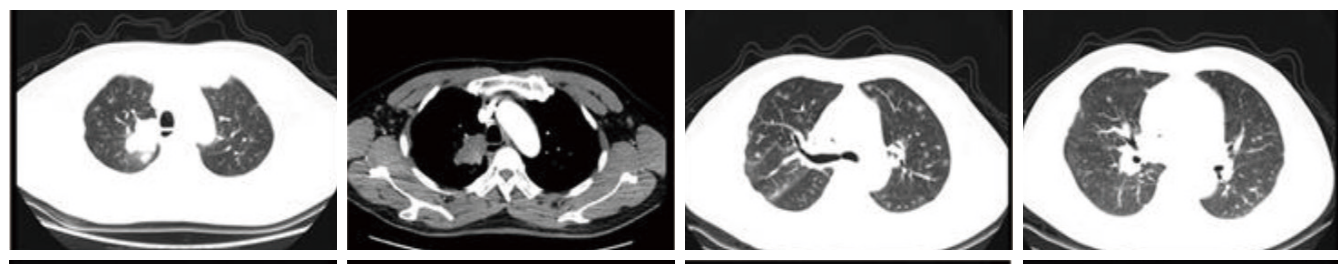

C

After the 2nd cycle of chemotherapy March 2018 (status: SD)
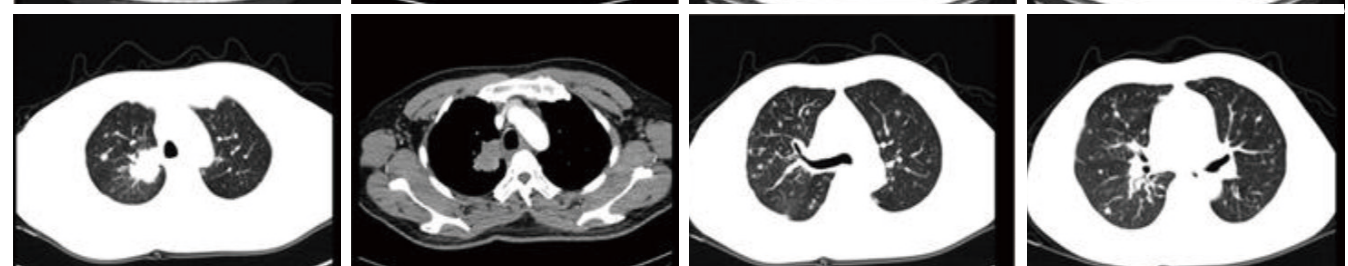

D

After the 4th cycle of chemotherapy May 2018 (status: PD)
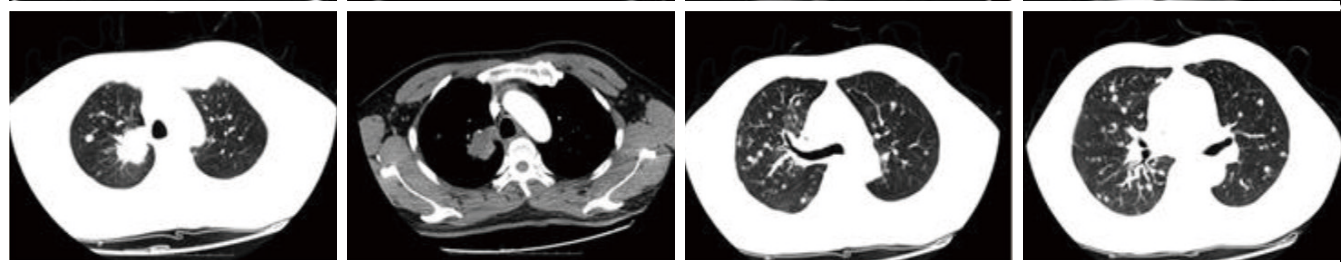

E
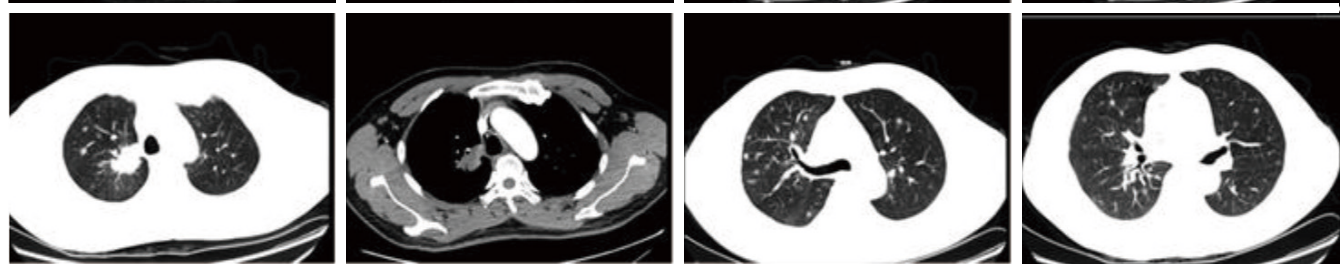

Figure 1 Chest CT scans before and after treatment. Chest computed-tomography scans of the patient from baseline (A), PD at 7 months after osimertinib therapy (B), SD after 2 cycles of chemotherapy regimen (C), PD after 4 cycles of chemotherapy regimen (D) and PR after 1 month of osimertinib rechallenge (E). PD, progressive disease; SD, stable disease; PR, partial response.

with the Helsinki Declaration (as revised in 2013). Written informed consent was obtained from the patient.

\section{Discussion}

EGFR-TKIs provide benefits to patients with EGFR sensitizing mutations even after developing resistance to previous EGFR-TKI therapy (8-12). However, information on rechallenge strategy has been limited to first and secondgeneration EGFR-TKIs. Successful rechallenge with first and second-generation EGFR-TKIs have demonstrated disease control rate of up to $85.2 \%$ and median PFS of up to 6 months (8). On the other hand, osimertinib, an irreversible inhibitor of EGFR sensitizing mutations as well as EGFR T790M, has demonstrated superior efficacy comparing to first and second-generation EGFRTKIs in patients with no EGFR T790M (6). Hence, osimertinib rechallenge in EGFR T790M-negative patients after EGFR-TKI failure is feasible and possibly a better treatment option. However, osimertinib readministration after EGFR-TKI failure in EGFR-T790Mnegative patients has not been reported. A case of successful osimertinib rechallenge in an EGFR T790M-positive NSCLC patient following osimertinib resistance then PD from chemotherapy-regimen has been reported to achieve PR (13). In addition, successful rechallenge with osimertinib has been reported in four T790M-positive NSCLC patients following development of resistance to abivertinib, another 


\begin{tabular}{lccccccccccc}
\hline AKT1 & ALK & APC & AR & ARID1A & ATM & ATR & B2M & BARD1 & BCL2L11 & BCOR & BLM \\
BRAF & BRCA1 & BRCA2 & BRINP3 & BRIP1 & CARD11 & CASP8 & CBL & CCND1 & CCNE1 & CD274 & CD74 \\
CDH18 & CDK4 & CDK6 & CDKN1A & CDKN1B & CDKN2A & CHEK1 & CHEK2 & CREBBP & CSMD3 & CTNNB1 & CYP2D6 \\
DIS3 & DNMT3A & DPYD & EGFR & EMSY & EP300 & EPHA3 & EPHA5 & EPHA7 & EPHB1 & ERBB2 & ERBB3 \\
ERBB4 & ESR1 & FANCA & FANCI & FAT3 & FBXW7 & FGF19 & FGF3 & FGF4 & FGFR1 & FGFR2 & FGFR3 \\
FLT1 & FLT3 & FLT4 & GATA2 & GATA3 & GRIN2A & H3F3C & HGF & HIST1H1C & HIST1H3B & HIST1H3G & HRAS \\
IDH1 & IDH2 & IGF2 & IKZF1 & IL7R & INHBA & JAK1 & JAK2 & KDM5A & KDM6A & KDR & KEAP1 \\
KIT & KMT2D & KRAS & LRP1B & MAP2K1 & MAP3K13 & MAX & MCL1 & MEN1 & MET & MLH1 & MRE11 \\
MSH2 & MSH6 & MTOR & MUTYH & MYC & MYCN & NAV3 & NBN & NF1 & NFE2L2 & NOTCH1 & NRAS \\
NRG1 & NTRK1 & NTRK2 & NTRK3 & PAK5 & PALB2 & PARP1 & PDGFRA & PDGFRB & PIK3C2G & PIK3C3 & PIK3CA \\
PIK3CG & PIK3R1 & PMS2 & POLD1 & POLE & POM121L12 & PPP2R1A & PRKDC & PTEN & PTPRD & PTPRT & RAD50 \\
RAD51B & RAD51C & RAD51D & RAD54L & RAF1 & RARA & RB1 & RBM10 & RET & RNF43 & ROS1 & RUNX1 \\
SETD2 & SMAD4 & SMARCA4 & SOX2 & SOX9 & SPOP & SPTA1 & SRC & STAG2 & STK11 & TBX3 & TERT \\
TGFBR2 & TP53 & TP63 & TRIM58 & TRPC5 & U2AF1 & UGT1A1 & VEGFA & VEGFB & VEGFC & VHL & YES1 \\
\hline
\end{tabular}

Figure 2 The 168 genes included in the OncoScreen Target panel (Burning Rock Biotech).

third-generation EGFR-TKI, achieving SD in 3 patients lasting 10, 12.8 and 14 months, respectively, and PR in a patient lasting for 7 months (14). Herein, we present a case of successful osimertinib rechallenge after osimertinib resistance in an EGFR 19del-positive, T790M-negative metastatic NSCLC patient. The favorable response with osimertinib rechallenge observed in our case was likely due to the increase in abundance of EGFR $19 \mathrm{del}$ clones resulting from the regrowth of $E G F R$ mutant clones and resensitization of the tumor cells to osimertinib. Our finding suggests that osimertinib re-administration can provide clinical benefit and should be considered as a therapeutic option for patients who have acquired osimertinib resistance with or without EGFR T790M.

A consequence of intratumoral heterogeneity is the diverse responses observed on the same treatment. In our current case, the detection of PIK3CA EQ545DK and TP53 V173L concurrent to EGFR 19del at baseline possibly may have potentially affected the initial response of our patient to gefitinib resulting in a PFS of only 7 months, which is at the lower range of the expected PFS for gefitinib-treated $E G F R$ mutant advanced NSCLC patients (Figure 3A) (18). Several studies have associated concurrent TP53 mutations with diminished sensitivity towards first and second-generation EGFRTKI, particularly in patients with EGFR 19del (19,20). In addition, activation of PIK3CA pathway, such as thru p110alpha mutation E545K, has been implicated as a primary resistance mechanism to EGFR-TKI $(21,22)$. Despite remarkable treatment response, all patients undergoing EGFR-TKI therapy will eventually acquire resistance and develop disease progression. A secondary mutation EGFR T790M, mediating about more than 50\% of resistance, can be targeted with the administration of third-generation EGFR-TKI $(4,5)$. In our case, EGFR T790M was only detected at an $\mathrm{AF}$ of $4.47 \%$ after treatment failure with gefitinib. Although undetected in succeeding sequencing analysis, the presence of EGFR T790M cannot be completely excluded due to intratumor heterogeneity and detection limit of the assay. Conversely, approximately $30 \%$ of resistance to third-generation EGFR-TKI is mediated by acquisition of EGFR C797S, with or without the loss of EGFR T790M mutation (23), such as the observed osimertinib resistance mediated by the acquisition of EGFR C797S in cis to T790M in the 4 cases reported by Zhang et al. (14). However, in our case, treatment failure with osimertinib did not involve EGFR C797S mutation but is likely to be mediated by CCDC6-RET fusion, which has been proposed as one of the resistance mechanisms to EGFR-TKIs $(24,25)$. Cabozantinib, a TKI targeting MET, RET, and VEGFR2 (26), was not administered due to limited access since it was not approved in China. CCDC6RET fusion has been first detected with an $\mathrm{AF}$ of $1.03 \%$ at $\mathrm{PD}$ after the first exposure to osimertinib, $\mathrm{AF}$ reduced 
A

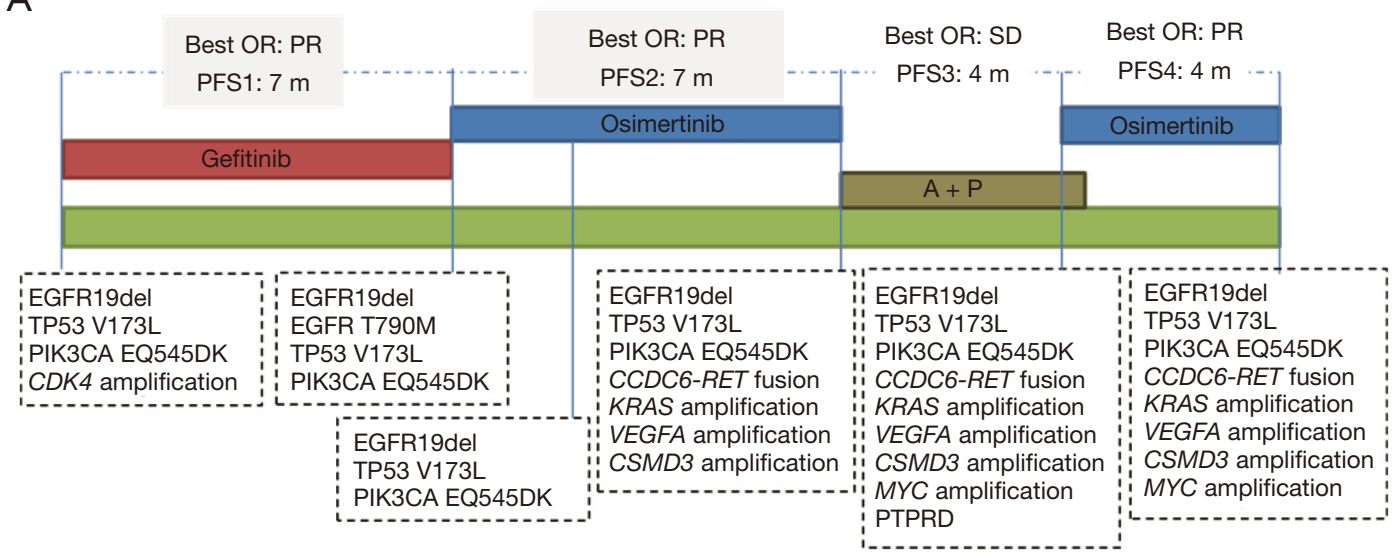

B

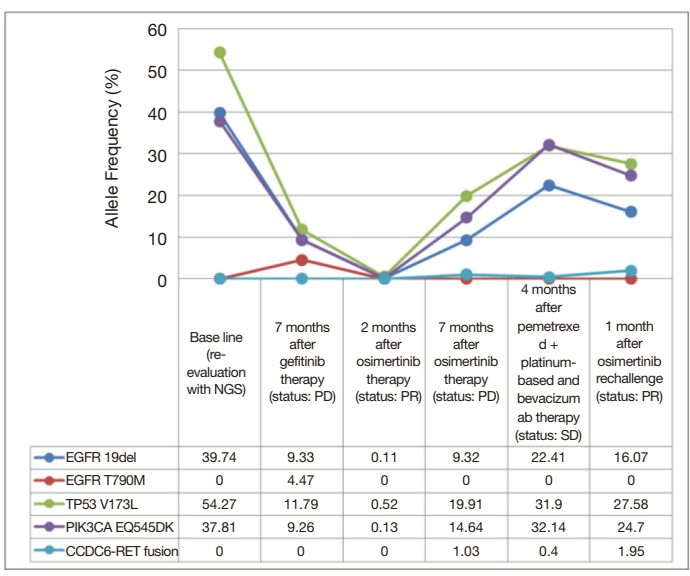

C

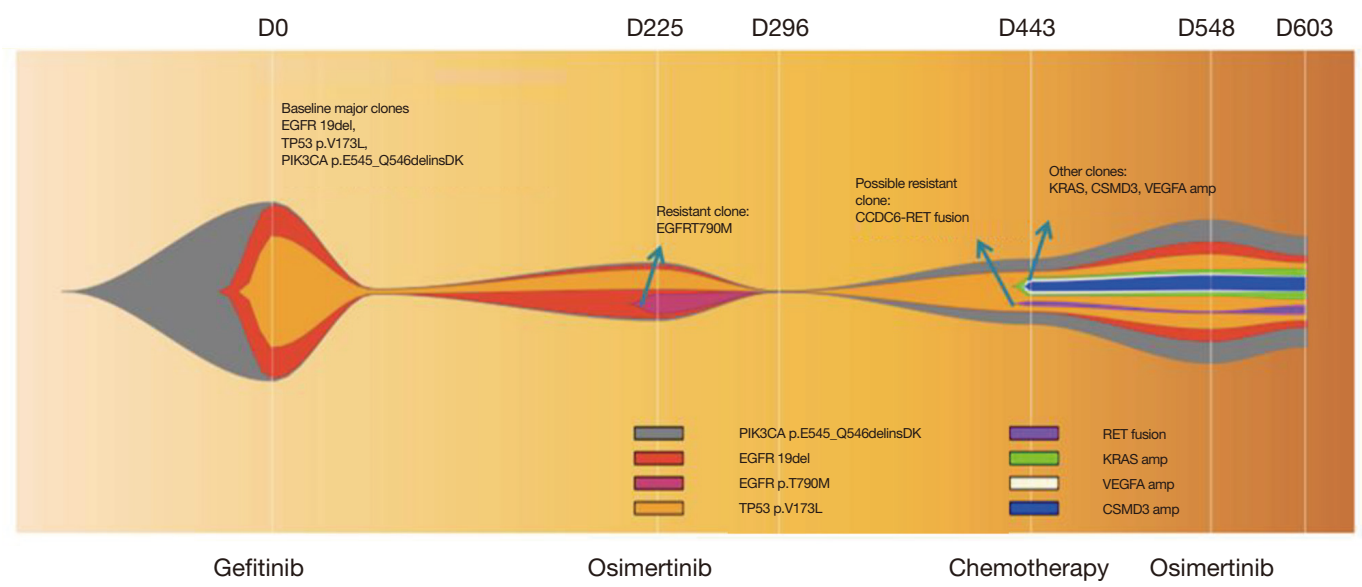

Figure 3 Genetic alterations detected in the patient. (A) Schematic diagram of the treatment received by the patient and genetic alterations detected at each time point. Best OR and PFS for each treatment (expressed in months) were also indicated. Highlighted genetic alterations were the basis for treatment choices. (B) The changes of allele frequencies of major genetic alterations detected. X-axis denotes the time point of sequencing. Y-axis denotes the allele frequency expressed in percentage. (C) Clonal evolution. Time points are indicated in days. Colors denote the different genetic alterations. Best OR, best overall objective response; PR, partial response; SD, stable disease; PFS, progression-free survival; $\mathrm{m}$, months; A+P, pemetrexed coupled with a platinum-based chemotherapy and bevacizumab; D, days. 
to $0.4 \%$ during the third-line chemotherapy regimen and again increased to $1.95 \%$ during the osimertinib rechallenge (Figure 3B). We postulate that CCDC6-RET fusion mediates osimertinib resistance in our case. The fish plot in Figure $3 C$ illustrates the clonal evolution in our patient in response to the treatment, depicting the possible resistant clones responsible for treatment failure.

Collectively, our finding suggests that osimertinib rechallenge can provide clinical benefit and should be considered as a therapeutic option for patients who have acquired osimertinib resistance regardless of EGFR T790M status. Targeted sequencing coupled with welldesigned panels catered for cancer diagnosis can provide a more comprehensive view on genetic alterations, which is invaluable for treatment guidance, monitoring as well as for understanding the resistance mechanism. Our case illustrates the importance of timely treatment monitoring.

\section{Acknowledgments}

We would like to thank Xiaojian Chen for his help in polishing the language of our paper.

Funding: None.

\section{Footnote}

Reporting Checklist: The authors have completed the CARE reporting checklist. Available at http://dx.doi. org/10.21037/apm-20-2369

Conflicts of Interest: All authors have completed the ICMJE uniform disclosure form (available at http://dx.doi. org/10.21037/apm-20-2369). The authors have no conflicts of interest to declare.

Ethical Statement: The authors are accountable for all aspects of the work in ensuring that questions related to the accuracy or integrity of any part of the work are appropriately investigated and resolved. All procedures performed in studies involving human participants were in accordance with the ethical standards of the institutional and national research committees and with the Helsinki Declaration (as revised in 2013). Written informed consent was obtained from the patient.

Open Access Statement: This is an Open Access article distributed in accordance with the Creative Commons Attribution-NonCommercial-NoDerivs 4.0 International
License (CC BY-NC-ND 4.0), which permits the noncommercial replication and distribution of the article with the strict proviso that no changes or edits are made and the original work is properly cited (including links to both the formal publication through the relevant DOI and the license). See: https://creativecommons.org/licenses/by-nc-nd/4.0/.

\section{References}

1. Mitsudomi T, Yatabe Y. Mutations of the epidermal growth factor receptor gene and related genes as determinants of epidermal growth factor receptor tyrosine kinase inhibitors sensitivity in lung cancer. Cancer Sci 2007;98:1817-24.

2. Yu HA, Arcila ME, Rekhtman N, et al. Analysis of tumor specimens at the time of acquired resistance to EGFR-TKI therapy in 155 patients with EGFR-mutant lung cancers. Clin Cancer Res 2013;19:2240-7.

3. Balak MN, Gong Y, Riely GJ, et al. Novel D761Y and common secondary T790M mutations in epidermal growth factor receptor-mutant lung adenocarcinomas with acquired resistance to kinase inhibitors. Clin Cancer Res 2006;12:6494-501.

4. Janne PA, Yang JC, Kim DW, et al. AZD9291 in EGFR inhibitor-resistant non-small-cell lung cancer. N Engl J Med 2015;372:1689-99.

5. Cross DA, Ashton SE, Ghiorghiu S, et al. AZD9291, an irreversible EGFR TKI, overcomes T790M-mediated resistance to EGFR inhibitors in lung cancer. Cancer Discov 2014;4:1046-61.

6. Soria JC, Ohe Y, Vansteenkiste J, et al. Osimertinib in Untreated EGFR-Mutated Advanced Non-Small Cell Lung Cancer. N Engl J Med 2018;378:113-25.

7. Mok TS, Wu YL, Ahn MJ, et al. Osimertinib or PlatinumPemetrexed in EGFR T790M-Positive Lung Cancer. N Engl J Med 2017;376:629-40.

8. Xia GH, Zeng Y, Fang Y, et al. Effect of EGFR-TKI retreatment following chemotherapy for advanced nonsmall cell lung cancer patients who underwent EGFRTKI. Cancer Biol Med 2014;11:270-6.

9. Tomizawa Y, Fujita Y, Tamura A, et al. Effect of gefitinib re-challenge to initial gefitinib responder with non-small cell lung cancer followed by chemotherapy. Lung Cancer 2010;68:269-72.

10. Hata A, Katakami N, Yoshioka H, et al. Erlotinib after gefitinib failure in relapsed non-small cell lung cancer: clinical benefit with optimal patient selection. Lung Cancer 2011;74:268-73.

11. Oh IJ, Ban HJ, Kim KS, et al. Retreatment of gefitinib in 
patients with non-small-cell lung cancer who previously controlled to gefitinib: a single-arm, open-label, phase II study. Lung Cancer 2012;77:121-7.

12. Cappuzzo F, Morabito A, Normanno N, et al. Efficacy and safety of rechallenge treatment with gefitinib in patients with advanced non-small cell lung cancer. Lung Cancer 2016;99:31-7.

13. Metro G, Baglivo S, Siggillino A, et al. Successful Response to Osimertinib Rechallenge after Intervening Chemotherapy in an EGFR T790M-Positive Lung Cancer Patient. Clin Drug Investig 2018;38:983-7.

14. Zhang YC, Zhou Q, Chen ZH, et al. The spatiotemporal evolution of EGFR C797S mutation in EGFR-mutant non-small cell lung cancer: opportunities for thirdgeneration EGFR inhibitors re-challenge. Science Bulletin 2019;64.

15. Miyauchi E, Ichinose M, Inoue A. Successful Osimertinib Rechallenge in a Patient with T790M-Mutant Non-Small Cell Lung Cancer after Osimertinib-Induced Interstitial Lung Disease. J Thorac Oncol 2017;12:e59-e61.

16. Yoshida H, Kim YH. Successful Osimertinib Rechallenge after Severe Osimertinib-Induced Hepatotoxicity. J Thorac Oncol 2017;12:e61-3.

17. Satoh S, Shiroyama T, Tamiya M, et al. Successful osimertinib rechallenge after osimertinib-induced pneumonitis in a patient with lung adenocarcinoma. Respir Med Case Rep 2018;23:68-70.

18. Kuan FC, Li SH, Wang CL, et al. Analysis of progressionfree survival of first-line tyrosine kinase inhibitors in patients with non-small cell lung cancer harboring leu858Arg or exon 19 deletions. Oncotarget 2017;8:1343-53.

19. Wang Z, Cheng Y, An T, et al. Detection of $<$ em $>$ EGFR $<$ / em> mutations in plasma circulating tumour DNA as a selection criterion for first-line gefitinib treatment in patients with advanced lung adenocarcinoma (BENEFIT): a phase 2, single-arm, multicentre clinical trial. Lancet Respir Med 2018;6:681-90.

20. Canale M, Petracci E, Delmonte A, et al. Impact of TP53 Mutations on Outcome in EGFR-Mutated Patients Treated with First-Line Tyrosine Kinase Inhibitors. Clin Cancer Res 2017;23:2195-202.

21. Morgillo F, Della Corte CM, Fasano M, et al. Mechanisms of resistance to EGFR-targeted drugs: lung cancer. ESMO Open 2016;1:e000060.

22. Engelman JA, Mukohara T, Zejnullahu K, et al. Allelic dilution obscures detection of a biologically significant resistance mutation in EGFR-amplified lung cancer. J Clin Invest 2006;116:2695-706.

23. Thress KS, Paweletz CP, Felip E, et al. Acquired EGFR C797S mutation mediates resistance to AZD9291 in nonsmall cell lung cancer harboring EGFR T790M. Nat Med 2015;21:560.

24. Klempner SJ, Bazhenova LA, Braiteh FS, et al. Emergence of RET rearrangement co-existing with activated EGFR mutation in EGFR-mutated NSCLC patients who had progressed on first- or second-generation EGFR TKI. Lung Cancer 2015;89:357-9.

25. Desai A, Segal J, Szeto L, et al. PS02.23 CCDC6-RET Fusion as a Mechanism of Acquired EGFR Resistance. J Thorac Oncol 2017;12:S1573.

26. Grullich C. Cabozantinib: a MET, RET, and VEGFR2 tyrosine kinase inhibitor. Recent Results Cancer Res 2014;201:207-14.
Cite this article as: Han ZJ, Luo N, Li L, Liu ZL. Successful osimertinib rechallenge following subsequent chemotherapy regimen in a patient with metastatic non-small cell lung carcinoma: a case report. Ann Palliat Med 2021;10(7):8413-8419. doi: 10.21037/apm-20-2369 